\title{
Transplanted Cells Are Essential for the Induction But Not the Expression of Cortical Plasticity
}

\author{
(-Mahmood S. Hoseini, ${ }^{1,2}$ - ${ }^{3}$ Benjamin Rakela, ${ }^{1,2}$ Quetzal Flores-Ramirez, ${ }^{3}{ }^{\circledR}$ Andrea R. Hasenstaub, ${ }^{1,4,5}$ \\ (ㄱ)Arturo Alvarez-Buylla, ${ }^{3,6}$ and ${ }^{\circledR}$ Michael P. Stryker ${ }^{1,2}$ \\ ${ }^{1}$ Center for Integrative Neuroscience, University of California, San Francisco, California 94158, ${ }^{2}$ Department of Physiology, University of California, \\ San Francisco, California 94143, ${ }^{3}$ Department of Neurological Surgery, University of California, San Francisco, California 94143, ${ }^{4}$ Coleman Memorial \\ Laboratory, University of California, San Francisco, California 94158, ${ }^{5}$ Department of Otolaryngology-Head and Neck Surgery, University of California, \\ San Francisco, California 94143, and ${ }^{6}$ The Eli and Edythe Broad Center of Regeneration Medicine and Stem Cell Research, University of California, \\ San Francisco, California 94143
}

Transplantation of even a small number of embryonic inhibitory neurons from the medial ganglionic eminence (MGE) into postnatal visual cortex makes it lose responsiveness to an eye deprived of vision when the transplanted neurons reach the age of the normal critical period of activity-dependent ocular dominance (OD) plasticity. The transplant might induce OD plasticity in the host circuitry or might instead construct a parallel circuit of its own to suppress cortical responses to the deprived eye. We transplanted MGE neurons expressing either archaerhodopsin or channelrhodopsin into the visual cortex of both male and female mice, closed one eyelid for 4-5 d, and, as expected, observed transplant-induced OD plasticity. This plasticity was evident even when the activity of the transplanted cells was suppressed or enhanced optogenetically, demonstrating that the plasticity was produced by changes in the host visual cortex.

Key words: interneuron transplantation; mouse; ocular dominance; plasticity; visual cortex

\section{Significance Statement}

Interneuron transplantation into mouse V1 creates a window of heightened plasticity that is quantitatively and qualitatively similar to the normal critical period; that is, short-term occlusion of either eye markedly changes ocular dominance (OD). The underlying mechanism of this process is not known. Transplanted interneurons might either form a separate circuit to maintain the OD shift or might instead trigger changes in the host circuity. We designed experiments to distinguish the two hypotheses. Our findings suggest that while inhibition produced by the transplanted cells triggers this form of plasticity, the host circuity is entirely responsible for maintaining the $\mathrm{OD}$ shift.

\section{Introduction}

Normal brain development is marked by temporally restricted windows of heightened experience-dependent plasticity known

Received June 18, 2019; revised July 25, 2019; accepted Aug. 1, 2019.

Author contributions: M.S.H. and M.P.S. designed research; M.S.H., B.R., and Q.F.-R. performed research; M.S.H. analyzed data; M.S.H. wrote the first draft of the paper; M.S.H., B.R., A.R.H., A.A.-B., and M.P.S. edited the paper; M.S.H. and M.P.S. wrote the paper; A.R.H. and A.A.-B. contributed unpublished reagents/analytic tools.

This work was supported by the National Institutes of Health (Grants R01EY025174 and R01DC014101). M.P.S. is a recipient of the Research to Prevent Blindness Stein Innovator Award. A.A.-B. is a Heather and Melanie Muss Endowed Chair and has been generously supported by the John G. Bowes Research Fund. A.R.H. has been supported by the Coleman Memorial Fund, Hearing Research, Inc., the Klingenstein Foundation, and the UCSF Program in Breakthrough Biomedical Research. We thank members of the Stryker, Hasenstaub, and Alvarez-Buylla laboratories for helpful discussions.

A.A.-B. is cofounder, serves on the scientific advisory board, and owns shares in Neurona Therapeutics. The remaining authors declare no competing financial interests.

Correspondence should be addressed to Michael P. Stryker at stryker@phy.ucsf.edu or Mahmood S. Hoseini at mhoseini@phy.ucsf.edu.

https://doi.org/10.1523/JNEUROSCl.1430-19.2019

Copyright $@ 2019$ the authors as critical periods (CPs). During a CP in primary visual cortex (V1) - postnatal day (P) 25-35 in mouse-changes in neuronal circuitry facilitate the matching of left eye and right eye receptive fields in V1 binocular neurons (Wang et al., 2010; for review, see Espinosa and Stryker, 2012). Occlusion of vision of either eye over this time, referred to as monocular deprivation (MD), prevents binocular matching and results in structural and functional changes that reduce neural responses to the deprived eye and increase them to the eye that remains open (Wiesel and Hubel, 1963). After the end of the CP, visual cortical plasticity in response to MD is qualitatively and quantitatively different: it consists mainly of an increase in responsiveness to the nondeprived eye with little or no reduction in deprived-eye responses and is much slower (Sato and Stryker, 2008).

Local inhibitory interneurons in V1 play a crucial role in opening the CP for ocular dominance (OD) plasticity, which begins $\sim 35 \mathrm{~d}$ after these neurons are generated in the medial ganglionic eminence (MGE) (Hensch et al., 1998; Hanover et al., 
1999; Pizzorusso et al., 2002). Once the CP has closed, V1 circuits are thought to remain stable throughout life. However, transplantation of newly generated parvalbumin or somatostatin interneurons from the MGE of a donor embryo into the V1 of a postnatal host opens a second brief $\mathrm{CP} \sim 35 \mathrm{~d}$ after transplantation (DAT) (Southwell et al., 2010; Tang et al., 2014; Davis et al., 2015). Similar transplantation has also shown promise in improving visual acuity in adult mice after long-term MD (Davis et al., 2015).

To dissect the precise contribution of inhibitory interneurons in OD plasticity, it is essential to draw a distinction between induction and expression of the OD shift. Induction of OD shift consists of a set of mechanisms (e.g., enhanced inhibition) that facilitate the occurrence and magnitude of the OD shift (Feldman, 2000). Conversely, expression refers to the changes in cortical circuit that maintain the reduced response to the deprived eye (Gandhi et al., 2008). Although inhibitory neuron activity is crucial in inducing the normal CP (Hensch et al., 1998; Kuhlman et al., 2013), the expression of the plasticity following MD no longer depends on inhibition (Saiepour et al., 2015). Here, we investigated whether the MGE transplants produce a second CP of OD plasticity by stimulating changes in host circuitry or, alternatively, by constructing a separate parallel circuit within the host tissue to inhibit deprived-eye responses and disinhibit nondeprived-eye responses. We transplanted inhibitory interneurons expressing either archaerhodopsin-3 or channelrhodopsin-2 that allowed us to silence or activate the transplanted interneurons after plasticity is induced by $4-5 \mathrm{~d}$ of MD during the second CP. Our results indicate that an OD shift induced by brief MD persists under conditions of either reduced or enhanced transplant-derived inhibition. Although manipulation of the activity of transplanted interneurons after plasticity has been induced alters the OD of some individual neurons, it does not change the overall OD at the population level. Our findings reveal that transplanted interneurons are not responsible for the expression of OD plasticity but rather facilitate its induction in the host circuitry.

\section{Materials and Methods}

Experimental animals. All protocols and procedures followed the guidelines of the Animal Care and Use Program at the University of California-San Francisco under Institutional Animals Care and Use Committee Protocol AN143347-03A. All mice were housed in standard conditions on a $12 \mathrm{~h}$ dark/light cycle. Experiments were performed during the dark, more active phase of the cycle. Ai40 (JAX Stock No: 021188), Ai32 (012569), Gad2-Cre (010802), and wild-type C57BL/6J (000664) lines were purchased from The Jackson Laboratory and maintained in our housing facility. This study was performed in strict accordance with the recommendations in the Guide for the Care and Use of Laboratory Animals of the National Institutes of Health.

Cell dissection and transplantation. The ventricular and subventricular zones of the MGE were dissected from E13.5 donor embryos as in Vogt et al. (2015). The tissue was dissociated by repeated pipetting in Leibovitz's L-15 medium containing 100U/ml DNase I (Sigma-Aldrich, D5025$15 \mathrm{KU})$. Dissociated cells were concentrated via centrifugation (800 rcf for $4 \mathrm{~min}$ ). P5-P8 recipients were anesthetized via hypothermia until pedal reflex disappeared and then placed on a stereotaxic platform for injection of concentrated cells ( $250-350$ cells/nl, $150 \mathrm{nl}$ per injection for $100-200 \mathrm{~K}$ cells total injected) through a beveled Drummond glass micropipette positioned 45 degrees from vertical. Three injections were placed into the caudal left cortex at $1.5 \mathrm{~mm}$ anterior from lambda, 3.5 $\mathrm{mm}$ lateral from midline and $1.5 \mathrm{~mm}$ anterior, $1 \mathrm{~mm}$ lateral, and $3 \mathrm{~mm}$ anterior, $1.25 \mathrm{~mm}$ lateral. Injections were made at a depth of $0.5-1.0 \mathrm{~mm}$ from the surface of the brain. After injection, recipients were placed on a heating pad until warm and active at which time they were returned to their mothers until weaning (P28). At the end of the experiment, brains were harvested, sliced, and imaged (see Fig. 1).

Head-plate surgery and alert recording apparatus. To allow for stable recordings, the animal's head was fixed to a rigid crossbar above the floating ball. This was achieved by attaching a titanium head plate (circular center with a $5 \mathrm{~mm}$ central opening) to the skull 1 week before the recording (28 DAT).

For head-plate attachment, animals were anesthetized with isoflurane in oxygen (3\% induction, $1.5 \%$ maintenance) and given a subcutaneous injection of Meloxicam (10 mg/kg) to reduce inflammation, a subcutaneous injection $(0.05 \mathrm{ml})$ of lidocaine above the skull as a local anesthetic, and a subcutaneous injection of $0.2 \mathrm{ml}$ of saline to prevent dehydration. After a scalp incision, the fascia was cleared from the surface of the skull and the titanium head plate was then attached with Metabond (Parkell), covering the entire skull, except for the region in the center of the head plate, which was covered with a $0.2 \%$ benzethonium chloride solution (New-Skin Liquid Bandage) to protect the skull. After recovery, the animal was allowed to habituate to the recording setup by spending $\sim 1 \mathrm{~h}$ on the floating ball over the course of $2-3 \mathrm{~d}$, during which time the animal was allowed to run freely. The polystyrene ball was constructed using two hollow 200-mm-diameter halves (Graham Sweet Studios) placed on a shallow polystyrene bowl ( $250 \mathrm{~mm}$ in diameter, $25 \mathrm{~mm}$ thick) with a single air inlet at the bottom. Two optical USB mice, placed $1 \mathrm{~mm}$ away from the edge of the ball, were used to sense rotation of the floating ball and transmit signals to our data analysis system using custom driver software. These measurements are used to divide data into still and running trials and analyze them separately.

Monocular deprivation and microelectrode recording. Four or $5 \mathrm{~d}$ before extracellular recording, monocular deprivation was induced by suturing the contralateral eyelid shut and was checked daily to confirm eyelid closure (see Fig. 1A).

Extracellular recording was performed in awake, head-fixed mice that were free to run on the floating polystyrene ball (see Fig. $2 A$ ). On the day of recording, the animal was anesthetized with isoflurane ( $3 \%$ induction, $1.5 \%$ maintenance) and sutures were removed to open the eyelid. The liquid bandage was removed from the skull, and a craniotomy of $\sim 1-2$ $\mathrm{mm}$ in diameter was made above the binocular zone of $\mathrm{V} 1$ (identified by intrinsic signal imaging). This small opening was large enough to allow for insertion of a 1.1-mm-long double-shank 128-channel electrode (Du et al., 2011), fabricated by the Masmanidis laboratory (University of California-Los Angeles) and assembled by the Litke laboratory (University of California-Santa Cruz). After animals recovered from anesthesia, the electrode was placed at an angle of $20-40^{\circ}$ to the normal of the cortical surface and inserted to a depth of $500-1000 \mu \mathrm{m}$. An optical fiber (400 $\mu \mathrm{m}$ diameter) coupled to a light source (green LED for archaerhodopsin, peak intensity $\sim 95 \mathrm{~mW} / \mathrm{mm}^{2}$ at $595 \mathrm{~nm}$; blue LED for channelrhodopsin, peak intensity $\sim 66 \mathrm{~mW} / \mathrm{mm}^{2}$ at $473 \mathrm{~nm}$ ) was positioned above the cranial window, centered on the binocular region, to illuminate V1. Recordings were started $1 \mathrm{~h}$ after electrode insertion. For each animal, the electrode was inserted not more than twice.

Visual stimuli. Stimuli were displayed on an LCD monitor (Dell, $30 \times$ $40 \mathrm{~cm}, 60 \mathrm{~Hz}$ refresh rate, $32 \mathrm{~cd} / \mathrm{m}^{2}$ mean luminance) placed $25 \mathrm{~cm}$ from the mouse $\left(-20^{\circ}\right.$ to $+40^{\circ}$ elevation $)$ without gamma correction. Drifting sinusoidal gratings at 12 evenly spaced directions (40 repetitions, $1.5 \mathrm{~s}$ duration, 0.04 cycles per degree, and $1 \mathrm{~Hz}$ temporal frequency) were generated and presented in random sequence using the MATLAB Psychophysics Toolbox (Brainard, 1997; Kleiner et al., 2007) followed by $1.5 \mathrm{~s}$ blank period of uniform $50 \%$ gray.

Data acquisition. Movement signals from the optical mice were acquired in an event-driven mode at up to $300 \mathrm{~Hz}$, and integrated at $100 \mathrm{~ms}$ intervals and then converted to the net physical displacement of the top surface of the ball. A mouse was said to be running on a single trial if its average speed for the first $500 \mathrm{~ms}$ of the trial fell above a threshold, found individually for each mouse $(1-3 \mathrm{~cm} / \mathrm{s})$, depending on the noise levels of the mouse tracker. Data acquisition was performed using an Intan Technologies RHD2000-Series Amplifier Evaluation System, sampled at $20 \mathrm{kHz}$; recording was triggered by a TTL pulse at the moment visual stimulation began. All data, code, and materials used in 
the analysis are available online at https://github.com/MahmoodHoseini/TransplantRoleODIExpression-JNeurosci.

Single-neuron analysis. Single units were identified using MountainSort (Chung et al., 2017), which allows for fully automated spike sorting of the data acquired using 128-site electrodes and runs at $\sim 2 \times$ real time. Manual curation after a run on $1 \mathrm{~h}$ of data takes an additional half hour, typically yielding 90 (range 50-130) isolated single units. Average waveforms of isolated single units were used to calculate three parameters by which cells were classified into narrow- or broad-spiking (Niell and Stryker, 2008). The parameters are as follows: the height of the positive peak relative to the negative trough, the slope of the waveform $0.5 \mathrm{~ms}$ after the negative trough, and the time from the negative trough to the peak (see Fig. 2B).

Mice were excluded under several conditions: (1) not enough transplanted cells observed in the binocular V1 $(n=7),(2)$ transplanted cells did not migrate to be able to establish proper connections with the host neurons $(n=3)$, or (3) the eyelid was not sutured properly $(n=1)$. Spikes from two or more units that could not be cleanly divided were classified as multiunit clusters and were excluded from further analysis. Running speed of the animal was used to divide trials into either a running or still state. For each single-unit, either the running or the still trials were used depending on whichever were more frequent. Cells with $<10$ trials were discarded.

Intrinsic signal imaging. After microelectrode recordings, OD of V1 was determined using intrinsic signal imaging (Cang et al., 2005). Mice were anesthetized with $0.7 \%$ isoflurane and a single i.p. injection of 2-5 $\mathrm{mg} / \mathrm{kg}$ chlorprothixene. The head plate was secured to a stereotaxic frame, filled with agarose, and topped with a coverslip to provide a flat imaging surface. Animals were maintained at $37.5^{\circ} \mathrm{C}$, measured using a rectal thermometer, and eyes were protected with silicone oil. Intrinsic signal images were acquired at 30 frames per second using a Dalsa 1M30 CCD camera with a red interference filter. The camera was focused 550 $\mu \mathrm{m}$ from the cortical surface and collected $610 \mathrm{~nm}$ light that was reflected off the visual cortex through the skull.

Visual response amplitude was defined as the average signal amplitude for each pixel and an OD index (ODI) was computed as $(C-I) /(C+I)$ where $C$ is the response to contralateral visual stimulation and $I$ is the response to ipsilateral visual stimulation (Cang et al., 2005).

Immunostaining. To determine whether transplanted cells were in the binocular zone of V1, animals were perfused transcardially after microelectrode recording first with ice-cold PBS and then with a solution of $4 \%$ paraformaldehyde (PFA) prepared in PBS. The brains were harvested and postfixed overnight in a solution of $4 \%$ PFA and subsequently cryoprotected by immersion in a PBS solution containing 30\% sucrose. The brains were sectioned coronally (40 $\mu \mathrm{m}$ thickness) using a sliding microtome (Leica). Free-floating sections were permeabilized in $0.25 \%$ Triton X-100/PBS solution, blocked for $1 \mathrm{~h}$ at room temperature in a blocking solution containing TBS, $10 \%$ normal goat serum, and $0.1 \%$ Triton X-100. They were then incubated overnight at $4 \mathrm{C}$ with 1:500 diluted chicken anti-GFP (Abcam, ab13970) primary antibody. After washing in PBS five times for $10 \mathrm{~min}$ each, sections were incubated in blocking solution with goat anti-chicken secondary antibodies (Thermo Fisher, Alexa Fluor 488, A-11039) at 1:1000 for $1 \mathrm{~h}$ at room temperature. They were then washed three times in PBS for 10 min each, mounted on glass slides using mounting media, and coverslipped.

Cell counting. Cell density was defined as the number of fluorescent cells within the binocular visual cortex divided by the total area. Binocular visual cortex was identified functionally during intrinsic signal imaging sessions. Fluorescent images of the binocular visual cortex were imaged using Nikon Eclipse 90i inverted microscope with a Nikon $4 \times$ objective.

Experimental design and statistical analysis. The experiments reported here were designed to determine whether optogenetic suppression/activation of the transplanted interneurons alters network activity and/or OD (Experiment 1) and if delivering the same optogenetic light affects network activity and/or OD in the control mice (Experiment 2). For Experiment 1, we recorded from 10 mice ( 6 females and 4 males) that were transplanted with interneurons expressing ArchT (suppression) and 5 mice ( 2 females and 3 males) that were transplanted with interneu- rons expressing ChR2 (activation). All data are illustrated in Figures 1, 3, 4, 5, 6, and 7 and Tables 1 and 2. For Experiment 2, we recorded from 4 mice ( 2 females and 2 males) that did not receive any transplant. All data are shown in the scatter plots of Figure 2. The main source of variability for all of these experiments is the individual neurons. Statistical significance was done in MATLAB and Python using a Mann-Whitney $U$, two-sample Kolmogorov-Smirnov, Wilcoxon rank-sum, Wilcoxon signed-rank test. Correction for multiple comparisons were done using the Benjamini-Hochberg procedure with a false discovery rate of 10\% (Benjamini and Hochberg, 1995) (https://www.mathworks.com/ matlabcentral/fileexchange/27418-fdr_bh). All data are presented as mean \pm SEM unless stated otherwise.

\section{Results}

Transplantation of MGE-derived interneurons into the visual cortex of adult mice induces a second CP of OD plasticity (Southwell et al., 2010). It is unknown what circuit changes are responsible for induction and maintenance of this form of OD plasticity. More specifically, is transplant-induced OD plasticity a consequence of changes in host circuitry like those in the normal CP, or is it instead the product of a parallel circuit constructed by the transplanted neurons themselves? To discriminate between the two alternative hypotheses, we transplanted MGE interneurons expressing either archaerhodopsin-3 (RCL-ArchT-EGFP) (Han et al., 2011) or channelrhodopsin-2 (RCL-ChR2-EYFP) (Madisen et al., 2012) to enable us to silence or activate them after plasticity was induced by $4-5 \mathrm{~d}$ of $\mathrm{MD}$ during the second $\mathrm{CP} \sim 33$ DAT (Fig. 1A).

Embryonic MGE-derived interneurons of age $13.5 \mathrm{~d}$ after gestation that express excitatory or inhibitory opsins were transplanted into the primary visual cortex of wild-type recipients. Transplanted interneurons dispersed and engrafted into the host cortex (Fig. $1 B$ ) and induced a second CP at 33 DAT revealed by $4 \mathrm{~d}$ of $\mathrm{MD}$ (Fig. $1 C$; average ODI, transplanted: mean $\pm \mathrm{SEM}=$ $0.24 \pm 0.03$ to $-0.01 \pm 0.01, p=0.01$, Wilcoxon signed-rank test, $n=8$ mice; control: $0.24 \pm 0.05$ to $0.18 \pm 0.03, p=0.07, n=$ 4 mice). Both experimental and control ODIs and OD shifts are consistent with the published data (Southwell et al., 2010; Saiepour et al., 2015; Larimer et al., 2016; Priya et al., 2019) and the absolute OD shift in all transplanted mice were larger than the corresponding measure in any of the control group $(p=0.004$, Wilcoxon rank-sum test). The overall density of transplanted cells in the host primary visual cortex at P46 was $109 \pm 9$ cells/ $\mathrm{mm}^{2}$ (Fig. 1D), and given the average neuronal density of $\sim 50,000$ cells $/ \mathrm{mm}^{2}$, transplanted cells constitute $\sim 5 \%$ of the number of interneurons in the host cortex. The number of transplanted cells across all the experimental mice was far more than the number that is required to induce the second CP (Tang et al., 2014; Larimer et al., 2016).

As the first control experiment, we confirmed that population activity measured with multisite microelectrodes reliably estimates OD. We performed extracellular recordings of binocular V1 zone of normally-reared, awake, head-fixed mice that did not receive any transplanted cells (Fig. 2A). Average spike waveforms were used to calculate three parameters by which cells could be classified unambiguously into narrow- or broad-spiking (Niell and Stryker, 2008) (Fig. 2B). Narrow-spiking cells are almost exclusively fast-spiking interneurons, whereas broad-spiking cells are $\sim 90 \%$ excitatory and $10 \%$ inhibitory cells (Connors and Kriegstein, 1986; Barthó et al., 2004; Atencio and Schreiner, 2008). Hereafter, for clarity, we refer to the narrow- and broadspiking units as inhibitory and excitatory neurons respectively. Recordings from normally-reared mice reveal that ODI of population activity results in values similar to what has been reported 

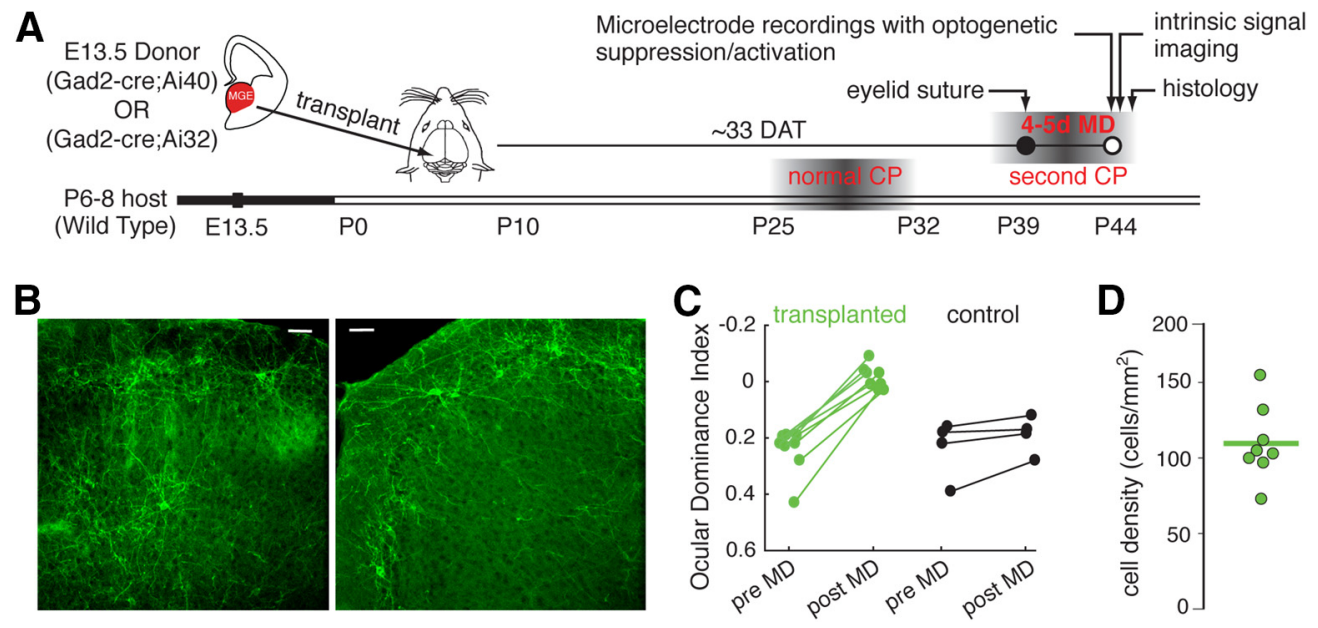

Figure 1. Transplanting medial ganglionic eminence (MGE)-derived interneurons induces a second CP. A, Cortical GABAergic interneurons are produced from E13.5 embryos of crossing Gad2-cre mouse line with either Ai40 (RCL-ArchT-EGFP) or Ai32 (RCL-ChR2-EYFP). Mouse visual cortex shows 1 week of heightened plasticity from P25 to P32, when inhibitory neurons are 33 to $35 \mathrm{~d}$ old. Similarly, inhibitory neuron precursors transplanted from E13.5 donor embryos into P6 -P8 hosts create a second CP from P38 - P45. Following 4-5 d of MD, visual responses were recorded using multisite microelectrodes in the presence of optogenetic manipulations. $\boldsymbol{B}$, Coronal brain sections, harvested after microelectrode recordings, at P46 show transplanted cells engrafted and functionally integrated in the binocular zone of the visual cortex. Scale bar, $50 \mu \mathrm{m}$. C, ODI showing significant plasticity following $4-5 \mathrm{~d}$ of MD in the transplant-recipient mice relative to the control animals as measured by optical imaging of intrinsic signal (transplanted: mean $\pm \mathrm{SEM}=0.24 \pm 0.03$ to $-0.01 \pm 0.01, p=0.01$, Wilcoxon signed-rank test, $n=8$ mice; control: $0.24 \pm 0.05$ to $0.19 \pm 0.03, p=0.07, n=4$ mice). $D, 0$ verall density of transplanted cells in the host primary visual cortex at P46 (109 \pm 9 transplant-derived neurons per mm ${ }^{2} ; n=8$ mice).

using intrinsic signal imaging (Fig. 2C; average ODI, $0.252 \pm$ $0.022, n=240$ ). In addition, our data indicates that inhibitory neurons respond more strongly to the deprived eye and have corresponding larger ODI (Gandhi et al., 2008) (Fig. 2C; average ODI, $0.18 \pm 0.03, n=132$ excitatory cells, 3 mice; $0.33 \pm 0.03$, $n=108$ inhibitory cells; $p=0.002$, Wilcoxon rank-sum test).

As the second control experiment, we tested for possible nonspecific light effects on the cortex of wild-type mice that did not receive any transplants but were subjected to $4 \mathrm{~d}$ of MD during the normal CP. Each eye's responses to visual stimuli were recorded with and without optogenetic light on interleaved visual stimulus trials. As expected, delivering the optogenetic stimulus light (green, $532 \mathrm{~nm}$ ) did not change network activity in either the excitatory (Fig. 2D) or inhibitory (Fig. $2 E$ ) cell population across all of the cortical layers (Fig. $2 F, G$ ). A small fraction of excitatory cells ( 3 of the 105) cells in these control animals were significantly modulated by the optogenetic stimulus light while visual stimuli were presented to the nondeprived eye (Benjamini-Hochberg adjusted $p=0.003 ; 4$ mice). Only 2 and 3 of 55 inhibitory cells displayed firing rate changes with optogenetics while visual stimuli were presented to nondeprived and deprived eyes, respectively (adjusted $p=0.020$ ). Not surprisingly, delivering optogenetic stimulus light to V1 did not alter ODI at the population-level of either cell type (Fig. $2 \mathrm{H}$, I; average ODI, light off to light on: $-0.062 \pm 0.038$ to $-0.071 \pm 0.041, n=105$ excitatory; $0.098 \pm 0.046$ to $0.112 \pm 0.047, n=55$ inhibitory cells, 4 mice).

As a final control experiment, we tested the effectiveness of optogenetic light in suppressing the activity of interneurons expressing ArchT. For this purpose, we recorded visual responses in a young donor mouse (strain Gad2-Cre;Ai40, P34) with and without optogenetic stimulation. In line with earlier studies (Cardin, 2012; Chuong et al., 2014; Fukunaga et al., 2014; Bukalo et al., 2015), optogenetic stimulation significantly reduces the activity in nearly all of the interneurons expressing ArchT (Fig. 3; average firing rate; light off, $23.5 \pm 4.2 \mathrm{~Hz}$; light on, $5.0 \pm 0.8 \mathrm{~Hz}$; $p=7 \mathrm{e}-4$, Wilcoxon signed-rank test, $n=17$ cells). These data demonstrate the efficacy of optogenetic activation of ArchT in suppressing neural activity.

Next, we tested whether optogenetic suppression of the transplanted interneurons was effective in altering network activity. Animals were transplanted with MGE-derived interneuron precursors expressing archaerhodopsin-3 (RCL-ArchT-EGFP) and were subjected to $4-5 \mathrm{~d}$ of $\mathrm{MD}$ during the transplant-mediated CP (Fig. 1A). Each eye's responses to visual stimuli were recorded with and without optogenetic perturbation of the activity of the transplanted interneurons on interleaved stimulus trials. Suppressing the activity of transplanted cells after they induce plasticity reduced the visual responses of many inhibitory neurons (Fig. 4A,B). Of 136 inhibitory neurons recorded, 55 and 23 were significantly modulated by the optogenetic stimulus light in response to nondeprived and deprived eyes ( $p=0.055$, BenjaminiHochberg adjusted, 63 cells show significant modulation in response to either eye; 10 mice). Most of the inhibitory neuron recordings are likely to have been from host neurons because the number of transplanted interneurons is very low and they disperse widely throughout the recipient tissue, making the probability of recording from them low.

Although optogenetic suppression of the transplants modulated inhibitory neuron activity in both directions, significant decreases were more common. The seemingly contradictory increases in inhibitory-cell firing rates are, however, expected in a balanced inhibition-stabilized network, and only a minority of cells is expected to show a detectable change in activity under perturbation (see Fig. 10 in Sadeh et al., 2017). Although reducing transplant-derived inhibition caused an increase in the firing rates of some excitatory cells, such enhanced excitation might activate other inhibitory cells (Moore et al., 2018). Figure $4 C$ shows that optogenetic suppression affected nondeprived-eye responses somewhat more strongly than it did deprived-eye responses. For the nondeprived eye, 21 cells were positively and 34 negatively modulated; while for the deprived eye, 6 and 17 cells, respectively, were positively and negatively modulated. Significant changes in the activity of interneurons were 
A
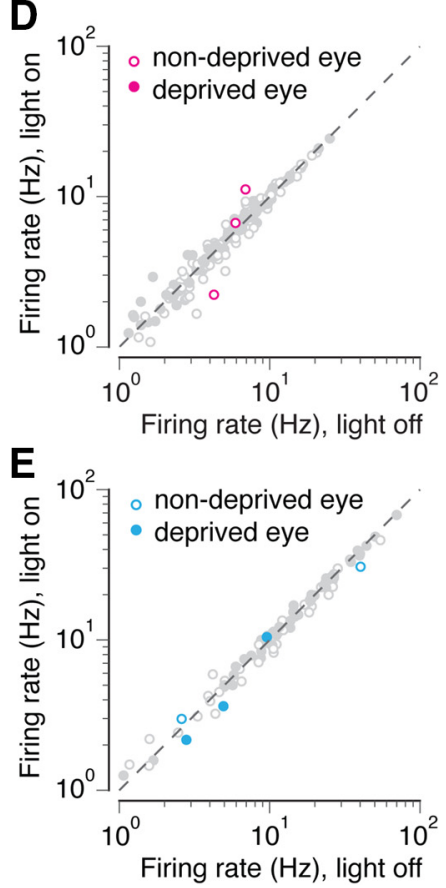

B

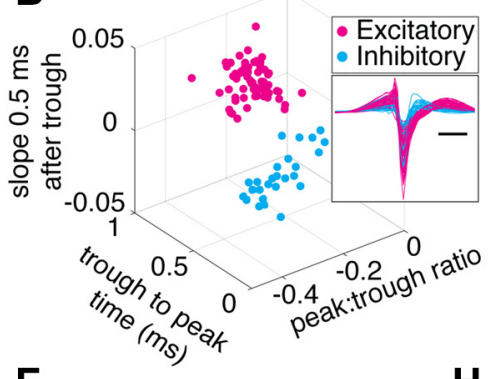

C

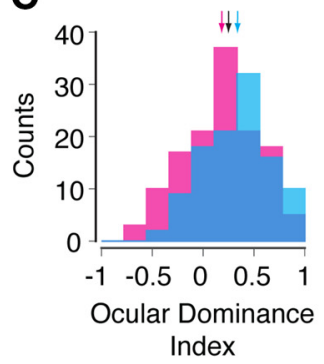

$F$

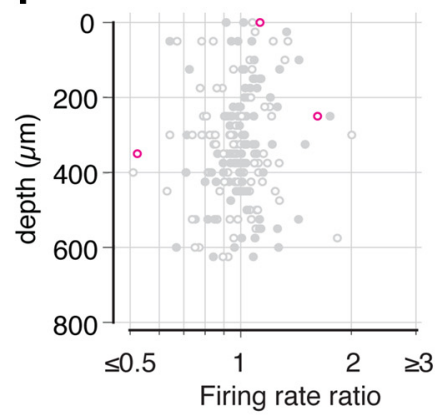

H

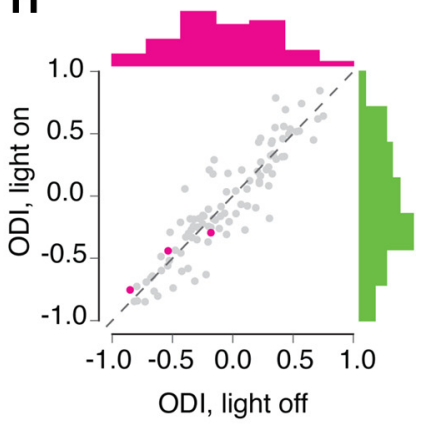

I

G

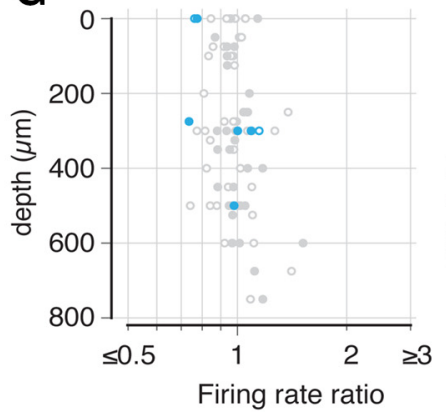

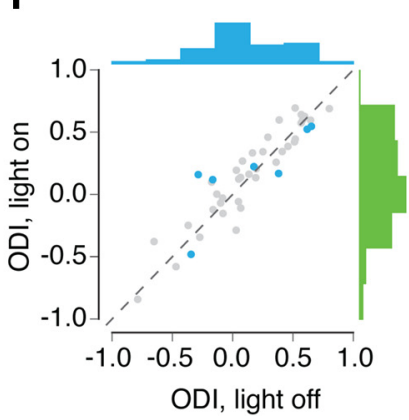

Figure 2. Optogenetic stimulation does not alter network activity and $0 \mathrm{DI}$ in the control mice. $A$, Recordings were acquired from the binocular visual cortex of freely moving mice while presented with drifting gratings ( 12 orientations, $1.5 \mathrm{~s}$ duration, and $1.5 \mathrm{~s}$ intertrial interval). $\boldsymbol{B}$, Using the three parameters calculated from average waveforms, cells were classified into narrow- (inhibitory, cyan) or broad- (excitatory, magenta) spiking (height of the positive peak relative to the negative trough: $-0.209 \pm 0.008,-0.287 \pm 0.018$, slope of the waveform $0.5 \mathrm{~ms}$ after the negative trough: $0.019 \pm 0.001,-0.011 \pm 0.001$, the time from the negative trough to the peak: $0.748 \pm 0.010,0.313 \pm 0.016 \mathrm{~ms}$, excitatory and inhibitory cells respectively). Shown in the subplot is the average spike waveforms for all units, aligned to minimum, demonstrating excitatory (magenta; 61) and inhibitory (cyan; 26) cells. C, The histogram of OD index across excitatory and inhibitory populations in control mice without MD (average ODI: black arrow, $0.252 \pm 0.022$, all cells; magenta arrow, $0.186 \pm 0.032, n=132$ excitatory cells, 3 mice; cyan arrow, $0.335 \pm 0.034, n=108$ inhibitory cells; $p=0.002$, Wilcoxon rank-sum test). $\boldsymbol{D}$, Evoked mean firing rate, averaged $>1 \mathrm{~s}$ window of all visual stimuli, of excitatory cells does not show any change from optogenetic stimulation (light on; green light, $532 \mathrm{~nm}$ ) to control (light off) condition for responses from both eyes (solid circles, deprived eye; open circles, nondeprived eye). Colored dots represent cells that show significant change in their firing rate. Of 105 excitatory cells, 3 and 0 cells are significantly modulated with optogenetic light while visual stimulus is presented to nondeprived (open circles) and deprived (solid circles) eyes (Benjamini-Hochberg adjusted $p=0.003 ; 4$ mice). Gray dashed line indicates unity. E, Same as in D for inhibitory cells. 0 f 55 inhibitory cells, 2 and 3 cells are significantly modulated with optogenetic light in response to nondeprived (open circles) and deprived (solid circles) eyes (Benjamini-Hochberg adjusted $p=0.020$ ). $\boldsymbol{F}$, Change in the ratio of firing rates against cortical depth for excitatory cells ( $\rho=-0.11, p=0.26$, Spearman rank-order correlation). $\boldsymbol{G}$, Same as in $\boldsymbol{F}$ for inhibitory cells $(\rho=0.10, p=0.43)$. $\boldsymbol{H}$, Scatterplots of $0 \mathrm{Dls}$ of excitatory cells under light-on versus light-off conditions. Distribution of $0 \mathrm{Dls}$ is shown as histogram on the top (light off, magenta) and right (light on, green) (light off to light on: $-0.062 \pm 0.038$ to $-0.071 \pm 0.041$, $p=0.43$, Wilcoxon signed-rank test; $n=105$ cells, 4 mice). $\boldsymbol{I}$, Same as in $\boldsymbol{H}$ for inhibitory cells (light off to light on: $0.098 \pm 0.046$ to $0.112 \pm 0.047, p=0.60 ; n=55$ cells).

observed across all cortical layers and in all 10 mice studied (Fig. 4D; Tables 1 and 2).

Optogenetic suppression of the activity of transplanted cells also affected the excitatory neurons, all of which were derived from the host (Fig. 5A). Again, the modulation of the excitatory populations was in both directions: the visual responses of some cells increased and that of others decreased, whereas the majority of the cells did not show any significant changes in their firing rates (Fig. 5B). Of 273 excitatory cells, 53 and 22 were significantly modulated by the optogenetic stimulus light in response to nondeprived and deprived eyes, respectively (Benjamini-Hochberg adjusted $p=0.029 ; 10$ mice). Significant increases were more common in excitatory cell population. In addition, optogenetic suppression caused a greater increase in nondeprived-eye than deprived-eye responses among excitatory cells across all cortical layers (Fig. 5C,D; Tables 1 and 2). Among the nondeprived-eye responses of excitatory neurons, 37 and 16 cells were positively and negatively modulated, respectively; for deprived-eye responses, 12 cells were positively and 10 cells negatively modulated.

Having verified that optogenetic stimulation was effective in suppressing the activity of transplanted cells, we then asked whether the plasticity produced by MD persisted when transplanted-cell activity was suppressed. First, we found that the OD shifts following $4 \mathrm{~d} \mathrm{MD}$ during the transplant-induced $\mathrm{CP}$ measured by single unit responses confirmed those measured by intrinsic signal imaging in that they were all significant but somewhat smaller than those following $\mathrm{MD}$ in the normal $\mathrm{CP}$ 

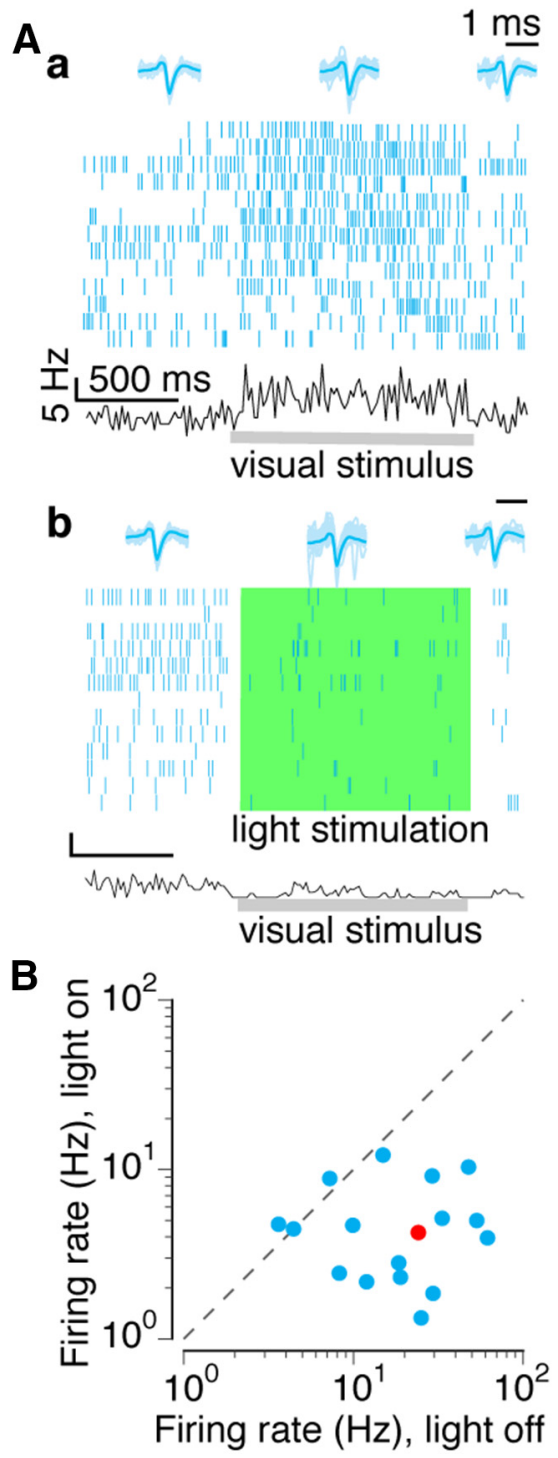

Figure 3. Optogenetic light effectively suppresses activity in the interneurons expressing ArchT. A, A representative inhibitory neuron expressing Arch T displays a significant decrease in firing rate with green light stimulation. $\boldsymbol{A} \boldsymbol{a}$, Spike raster for a representative inhibitory neuron across 13 trials with its instantaneous firing rate shown at the bottom ( $20 \mathrm{~ms}$ bins). Shaded area shows the duration of the stimulus. Shown at the top are the individual (light) overlaid with average (dark) waveforms for epochs before, during, and after stimulus presentation. $\boldsymbol{A} \boldsymbol{b}$, Same as in $\boldsymbol{A a}$ with optogenetic silencing. Green shading shows the duration of optogenetic suppression (green light, $532 \mathrm{~nm}$ ). $\boldsymbol{B}$, Evoked mean firing rate, averaged over visual stimulus duration, of inhibitory cells with optogenetic suppression (light on) against control (light off) (average firing rate; light off, $23.5 \pm 4.2 \mathrm{~Hz}$; light on, $5.0 \pm 0.8 \mathrm{~Hz} ; p=7 \mathrm{e}-4$, Wilcoxon signed-rank test, $n=17$ cells). Gray dashed line indicates unity. Red dot indicates the example cell shown in $\boldsymbol{A}$.

(average ODI after MD; Fig. 2, normal CP, $-0.006 \pm 0.030, n=$ 160 cells; Fig. $6 A, B$, second CP, $0.102 \pm 0.015, n=578$ cells; $p=$ 0.0008 , Wilcoxon signed-rank test). Second, we found that suppressing transplanted cells did not cause a consistent change in the ODIs of either the excitatory cells (Fig. 6A; average ODI, optogenetic excitation light off to light on: $0.067 \pm 0.019$ to $0.046 \pm 0.019, p=0.12$, Wilcoxon signed-rank test; $n=273$ cells, 10 mice) or the inhibitory cells (Fig. $6 B$; average ODI, light off to light on: $0.189 \pm 0.024$ to $0.166 \pm 0.027, p=0.12 ; n=136$ cells, 10 mice). Although the ODI had certainly been shifted by the deprivation from control values (Fig. $6 C$ ), the shift persisted under optogenetic suppression. Optogenetic suppression caused no
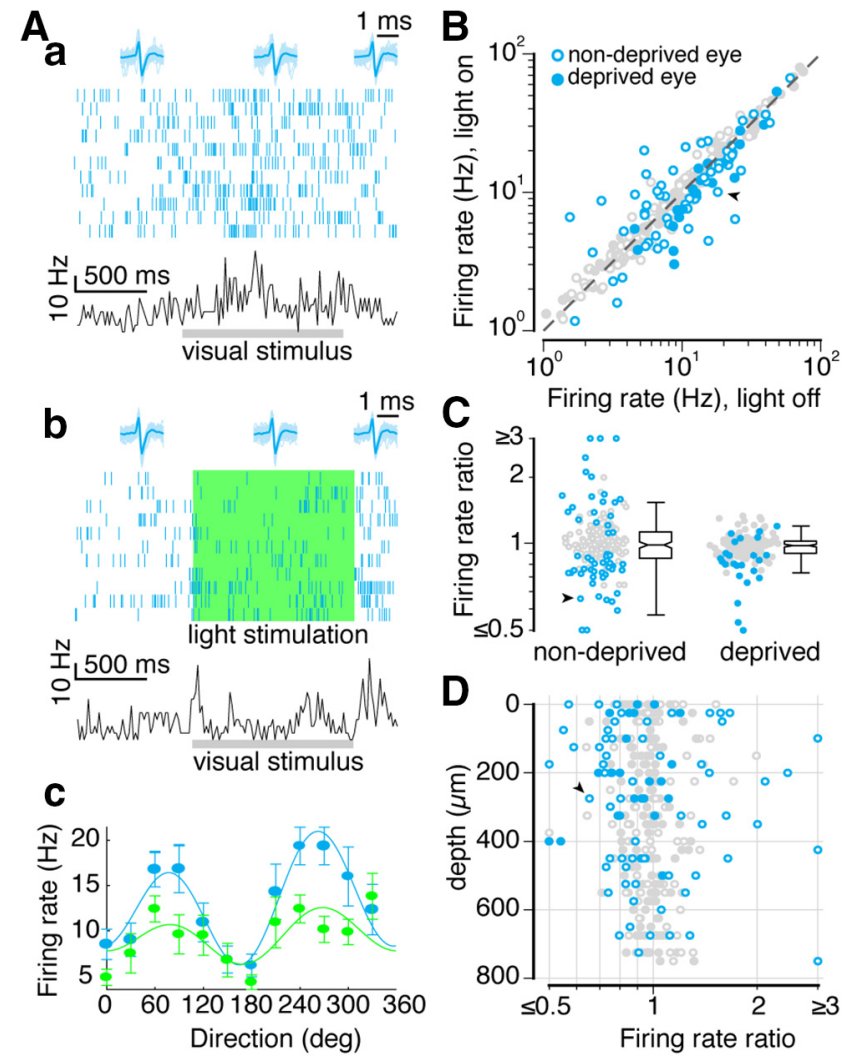

Figure 4. Suppressing MGE-derived transplanted interneurons reduces interneuron population activity across different cortical depths. $\boldsymbol{A}$, An example inhibitory cell that shows a decrease in firing rate with optogenetic suppression of transplanted cells. Aa, Spike raster for a representative inhibitory neuron across trials with its instantaneous firing rate shown at the bottom ( $20 \mathrm{~ms}$ bins). Shaded area shows the duration of the stimulus. Shown at the top are the individual (low opacity) overlaid with average (high opacity) waveforms for epochs before, during, and after stimulus presentation. $\boldsymbol{A} \boldsymbol{b}$, Same as in $\boldsymbol{A} \boldsymbol{a}$ with optogenetic silencing. Green shaded shows the duration of optogenetic suppression. Ac, Tuning curves for evoked responses in control (cyan) and optogenetic suppression (green) conditions. Solid curves are fit to a sum of two sinusoids. $\boldsymbol{B}$, Evoked mean firing rate, averaged over all visual stimuli for a $1 \mathrm{~s}$ window following stimulus onset, of inhibitory cells with optogenetic suppression (light on) against control (light off) in responses recorded from both eyes (slope $=0.97, r=0.97$ ). Colored dots represent cells that have significant firing rate modulation. Of 136 inhibitory cells, 55 and 23 cells are significantly modulated with optogenetic silencing in response to nondeprived (open circles) and deprived (solid circles) eyes (Benjamini-Hochberg adjusted $p=0.055$ ), 63 cells show significant modulation in either eye responses ( 10 mice). Gray dashed line indicates unity. Arrowhead indicates the example cell shown in A. C, The ratio of light on to light off average firing rates of inhibitory neurons for nondeprived-(left, 21 and 34 cells positively and negatively modulated, respectively; $1.077 \pm 0.003$ ) and deprived- (right, 6 and 17 cells positively and negatively modulated, respectively; $0.971 \pm 0.002$ ) eye responses. $\boldsymbol{D}$, Change in the ratio of mean firing rates against cortical depth, measured from the top channel in the brain, for inhibitory cells $(\rho=0.01, p=0.89$, Spearman rank-order correlation).

Table 1. Number of cells significantly modulated by optogenetic manipulation of transplanted cells in response to nondeprived eye

\begin{tabular}{llllllllllll}
\hline Mouse no. & 1 & 2 & 3 & 4 & 5 & 6 & 7 & 8 & 9 & 10 & Total \\
\hline Sex & $\mathrm{M}$ & $\mathrm{F}$ & $\mathrm{F}$ & $\mathrm{F}$ & $\mathrm{F}$ & $\mathrm{M}$ & $\mathrm{M}$ & $\mathrm{F}$ & $\mathrm{F}$ & $\mathrm{M}$ & \\
Excitatory & $3 / 12$ & $7 / 31$ & $9 / 42$ & $7 / 37$ & $4 / 27$ & $4 / 17$ & $6 / 42$ & $6 / 42$ & $4 / 20$ & $3 / 21$ & $53 / 273$ \\
Inhibitory & $2 / 4$ & $8 / 20$ & $7 / 25$ & $8 / 17$ & $5 / 15$ & $4 / 12$ & $11 / 21$ & $3 / 8$ & $4 / 8$ & $3 / 6$ & $55 / 136$ \\
\hline
\end{tabular}

Table 2. Number of cells significantly modulated by optogenetic manipulation of transplanted cells in response to deprived eye

\begin{tabular}{llllllllllll}
\hline Mouse no. & 1 & 2 & 3 & 4 & 5 & 6 & 7 & 8 & 9 & 10 & Total \\
\hline Sex & $\mathrm{M}$ & $\mathrm{F}$ & $\mathrm{F}$ & $\mathrm{F}$ & $\mathrm{F}$ & $\mathrm{M}$ & $\mathrm{M}$ & $\mathrm{F}$ & $\mathrm{F}$ & $\mathrm{M}$ & \\
Excitatory & $1 / 12$ & $2 / 31$ & $2 / 42$ & $4 / 37$ & $3 / 27$ & $0 / 17$ & $4 / 42$ & $0 / 24$ & $4 / 20$ & $2 / 21$ & $22 / 273$ \\
Inhibitory & $0 / 4$ & $3 / 20$ & $3 / 25$ & $3 / 17$ & $2 / 15$ & $3 / 12$ & $4 / 21$ & $2 / 8$ & $1 / 8$ & $2 / 6$ & $23 / 136$ \\
\hline
\end{tabular}


$A_{a}$
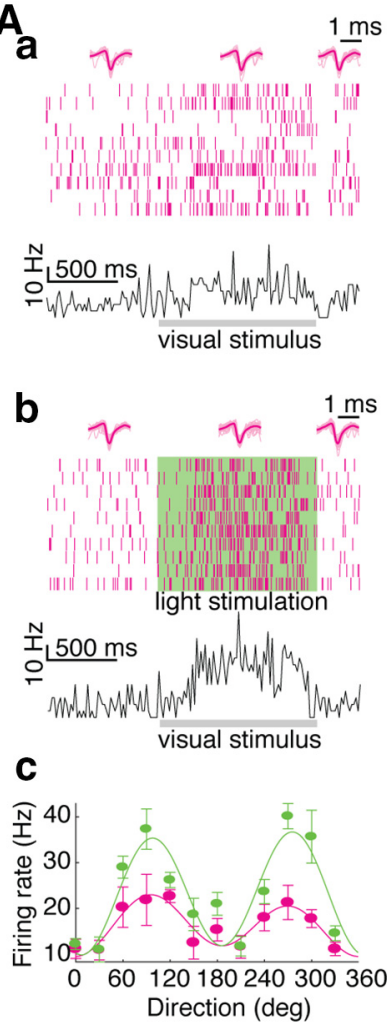

B

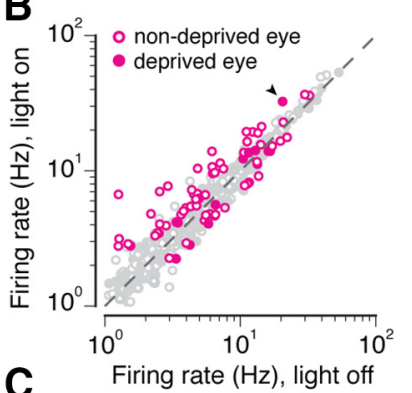

C Firing rate $(\mathrm{Hz})$, light off $\quad$ B
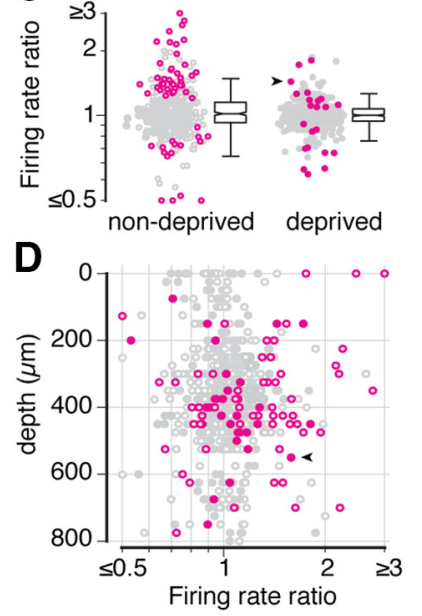

Figure 5. Suppressing MGE-derived transplanted interneurons increases excitatory population activity across different cortical depths. A, An example excitatory cell that shows an increase in firing rate with optogenetic suppression of transplanted cells presented in the same format as Figure $3 A$. B Evoked mean firing rate, averaged over all visual stimuli for a $1 \mathrm{~s}$ window following stimulus onset, of excitatory cells for light on against light off condition in both eye responses (slope $=1.03, r=0.98$ ). Colored dots represent cells that have significant firing rate modulation. 0 f 273 excitatory cells, 53 and 22 cells are significantly modulated with optogenetic light in response to nondeprived (open circles) and deprived (solid circles) eyes (Benjamini-Hochberg adjusted $p=0.029$ ), 66 cells show significant modulation in response to either eye stimulation (10 mice). Gray dashed line indicates unity. Arrowhead indicates the example cell shown in A.C, The ratio of light on to light off average firing rates of excitatory neurons for nondeprived- (left, 37 and 16 cells positively and negatively modulated, respectively; $1.065 \pm 0.001)$ and deprived- (right, 12 and 10 cells positively and negatively modulated, respectively; $1.013 \pm$ 0.001 ) eye responses. $D$, Change in the ratio of mean firing rates against cortical depth, measured from the top channel in the brain, for excitatory cells $(\rho=0.09, p=0.08$, Spearman rank-order correlation).

consistent change even in the subset of cells that were significantly affected by the optogenetic stimulus $(p=0.3$, excitatory; $p=0.59$, inhibitory; Wilcoxon rank-sum test), consistent with previous reports in the normal CP (Khibnik et al., 2010; Saiepour et al., 2015). The difference of OD distributions between excitatory and inhibitory cells raises the possibility that they may show different plasticity. However, the cumulative distributions of ODI indicate that the inhibitory cells continued to be more biased toward the deprived eye than the excitatory cells as they are in control animals, showing that the two populations are more or less similarly affected by MD (Fig. $6 C$; light off, $p=0.002$; light on, $p=0.005$; two-sample Kolmogorov-Smirnov test). Consistent with the fact that the optogenetic stimulation affects activity across all cortical depth (Figs. 4D, 5D), no consistent relationship was observed between changes in ODI and cortical depths (Fig. $6 D$; excitatory, $\rho=0.05, p=0.26$; inhibitory, $\rho=0.09, p=0.23$; Spearman rank-order correlation).

An alternative approach to determine whether transplantation specifically inhibits deprived-eye responses after MD is to

A
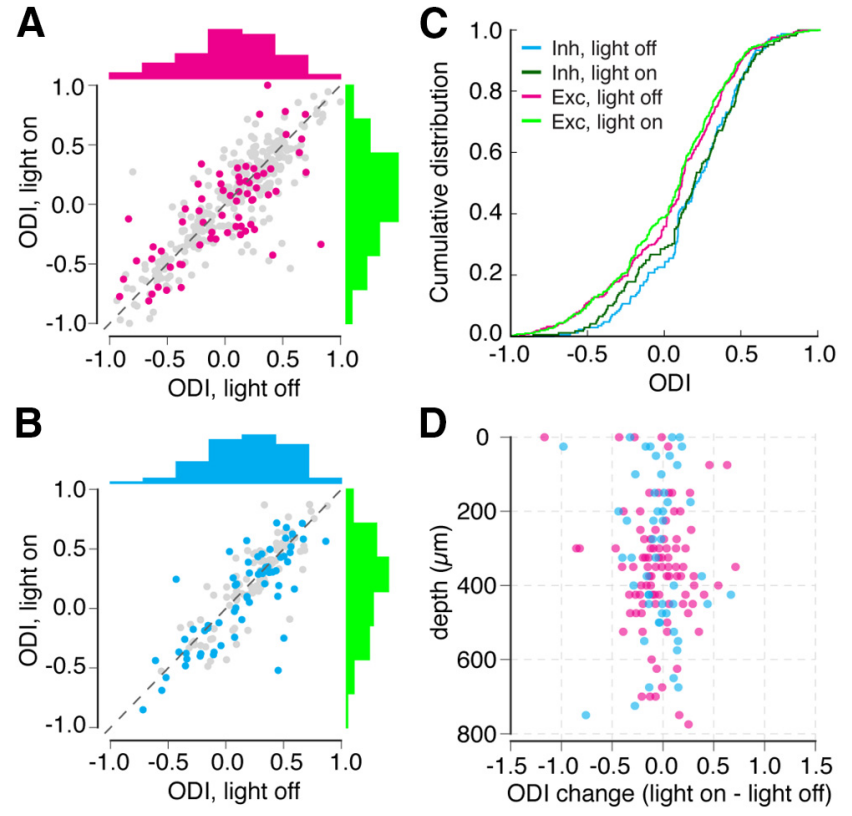

Figure 6. Excitatory and inhibitory population-level ODIs are not altered by suppressing transplanted interneurons. $\boldsymbol{A}$, Scatterplots of ODIs of excitatory cells under light on versus light off conditions. Distribution of ODIs is shown as histogram on the top (light off, magenta) and right (light on, green) (light off to light on: $0.067 \pm 0.019$ to $0.046 \pm 0.019, p=0.12$, Wilcoxon signed-rank test; $n=273$ cells, 10 mice). $B$, Same as in $\boldsymbol{A}$ for inhibitory cells (light off to light on: $0.189 \pm 0.024$ to $0.166 \pm 0.027, p=0.12 ; n=136$ cells, 10 mice). C, Cumulative distribution for the excitatory and inhibitory cells with and without optogenetic silencing (light on vs light off; magenta vs light green, excitatory, $p=0.86$; cyan vs dark green, inhibitory, $p=$ 0.95; two-sample Kolmogorov-Smirnov test). D, Change in the ODI against cortical depth for excitatory cells (magenta) and inhibitory cells (cyan) (excitatory, $\rho=0.05, p=0.26$; inhibitory, $\rho=0.09, p=0.23$; Spearman rank-order correlation).

activate the transplanted cells. Since optogenetic activation and inactivation of interneurons need not produce symmetric effects (Phillips and Hasenstaub, 2016; Moore et al., 2018), the outcome of this experiment is not trivial. To activate transplanted cells optogenetically, donor embryos expressing the gene for channelrhodopsin-2 in inhibitory neurons were generated by crossing Gad2-Cre mouse line with Ai32 (RCL-ChR2-EYFP) line. After transplantation of MGE neurons at P6 and 4-5 d of MD $35 \mathrm{~d}$ thereafter, recordings similar to those in which the transplanted cells carried an inhibitory opsin were performed (Fig. 1A). As expected, activating the transplanted interneurons altered activity in both cell types and across cortical depths. Of 405 excitatory cells, 76 and 61 were significantly modulated by the optogenetic stimulus light in response to nondeprived and deprived eyes, respectively (Fig. 7A; 95 cells show significant modulation in response to either eye; Benjamini-Hochberg adjusted $p=0.021,4$ mice). Of 244 inhibitory cells, 52 and 48 were significantly modulated by the optogenetic stimulus light in response to nondeprived and deprived eyes, respectively (Fig. 7B; 79 cells show significant modulation in response to either eye; Benjamini-Hochberg adjusted $p=0.018 ; 4$ mice). However, the optogenetic activation did not consistently favor one eye over the other, and the shifted ODI did not significantly change for either excitatory or inhibitory cells (Fig. 7C-F; excitatory, ODI change, $0.005 \pm 0.010, p=0.76$, Wilcoxon signed-rank test, $n=405$ cells from 5 mice; inhibitory, $-0.007 \pm 0.013, p=0.57 ; n=244$ ). Together, these findings confirm the hypothesis that the activity of transplanted cells is not required for the expression of OD plasticity, indicating rather that transplantation induces plasticity in the host circuit. 


\section{Discussion}

How plausible is that a small number of transplanted interneurons, typically $10 \%$ of the number of interneurons in the host tissue, could form a parallel circuit to directly inhibit the responses to the deprived eye? Because excitation and inhibition are finely balanced in the V1 cortical network (Troyer et al., 1998) an increase of the inhibition of the deprived eye's pathway by $10 \%$ would be expected to reduce its response substantially and could account for the OD shift. However, our findings reveal that transplanted neurons do not form a parallel circuit to mediate the expression of OD shift; rather they facilitate changes in the host circuit.

How do underlying neuronal mechanisms of the transplant-induced CP compare to the normal $\mathrm{CP}$ in developing visual cortex? The similarities can be recapitulated as follows: First, it has been shown previously that the maturation of GABAergic inhibitory circuits is essential for the induction of the normal $\mathrm{CP}$ (Hensch, 2005; Espinosa and Stryker, 2012). Similarly, the timing of transplantinduced $\mathrm{CP}$ is dictated by the maturation of the transplanted cells (Southwell et al., 2010). Second, optogenetic suppression of host interneurons alters cortical responses but does not affect the OD of the population after MD during the normal CP (Saiepour et al., 2015), indicating that the plasticity induced during the normal $\mathrm{CP}$ is not a selective inhibition of deprived eye pathways and disinhibition of pathways serving the nondeprived eye. Excitatory pathways are modified during the normal CP (Espinosa and Stryker, 2012). The present findings suggest a similar mechanism for transplant-induced OD plasticity. Our findings imply that OD plasticity in a property of the host network and not merely of the transplanted inhibitory cells.

If the endogenous inhibitory circuit is compromised by a reduction in GABA release, the normal CP does not start until it is rescued by pharmacological enhancement of GABAergic transmission (Hensch et al., 1998; Fagiolini and Hensch, 2000). Likewise, transplanted interneurons that lack the vesicular GABA transporter (and consequently fail to inhibit the neurons that they contact) fail to induce a second CP (Priya et al., 2019), despite the fact that they appear to develop similarly to wild-type neurons in that they disperse broadly in the host visual cortex, retain normal anatomical and electrophysiological properties, and receive effective synaptic inputs. Their failure to induce a second CP indicates that the output of these cells is essential for the plasticity to be reinstated.

Specific changes in the activity of fast-spiking, parvalbuminpositive basket interneurons have been shown to regulate the normal CP for OD plasticity (Kuhlman et al., 2011; Hengen et al., 2013; Figueroa Velez et al., 2017). However, either parvalbuminor somatostatin-positive cells can induce a second CP after transplantation into the visual cortex (Tang et al., 2014). Recent findings on the development of somatostatin-positive cells raise
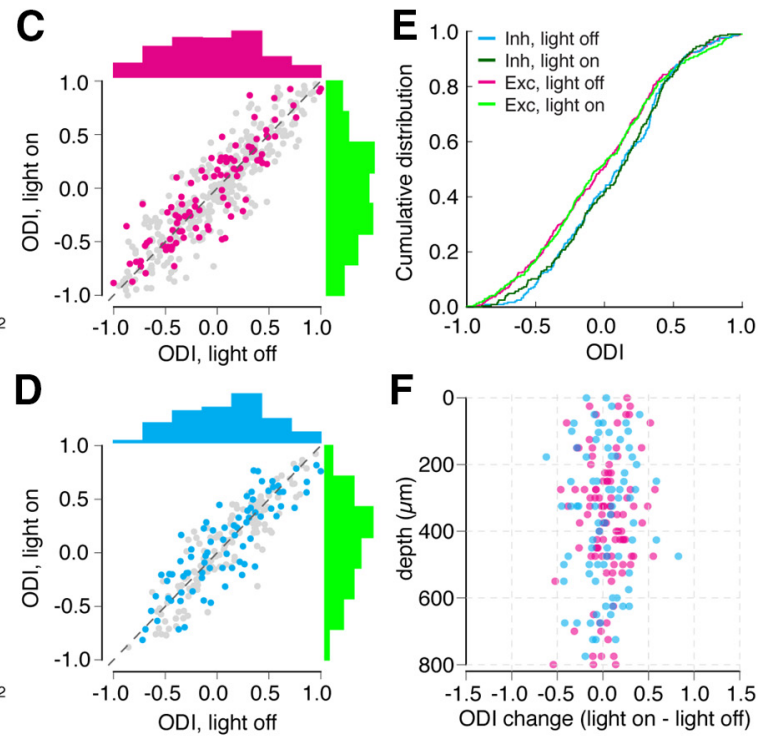

Figure 7. Excitatory and inhibitory population-level ODIs are not altered by activating transplanted interneurons. $\boldsymbol{A}$, Evoked (light on) against control (light off) in responses recorded from both

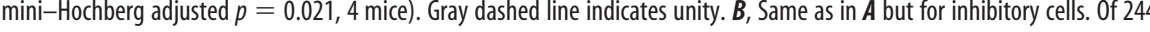
esectively (79 cells show significant modulation in response to either eye; Benjamini-Hochberg adjusted $p=0.018 ; 4$ micer (magenta, excitatory; cyan, inhibitory) (excitatory, $p=0.97$; inhibitory, $p=0.92$; two-sample Kolmogorov-Smirnov test). $\boldsymbol{F}$ Change in the 0DI against cortical depth for excitatory cells (magenta) and inhibitory cells (cyan) (excitatory, $\rho=-0.08, p=0.11$; inhibitory, $\rho=0.05, p=0.43$; Spearman rank-order correlation).

the possibility that they too may regulate the normal CP (Yaeger et al., 2019). These findings all point to a unique role for MGE-derived interneurons in triggering subsequent competitive plasticity in excitatory connections. CGE-derived interneurons by contrast are incompetent to induce OD plasticity (Larimer et al., 2016).

Adult OD plasticity is qualitatively and quantitatively different from CP OD plasticity (Espinosa and Stryker, 2012). First, CP plasticity is faster, stronger, and more persistent; a short $\mathrm{MD}$ is enough to induce an observable shift in OD while longer MD is required to cause comparable OD shift in adult mice (Sawtell et al., 2003). Second, the OD shift in adults induced is predominantly mediated by an increase in open eye responses with only a small decrease in deprived-eye responses (Sawtell et al., 2003; Hofer et al., 2006; Sato and Stryker, 2008). In contrast, OD plasticity during $\mathrm{CP}$ incorporates a strong decrease in deprived-eye responses followed by a significant increase in nondeprived-eye responses (for review, see Espinosa and Stryker, 2012). A number of recent studies have disclosed manipulations that activate and/or preserve CP-like plasticity in adult animal, including sensory-motor interaction (Kaneko and Stryker, 2014), social experience (Balog et al., 2014), environmental enrichment (Greifzu et al., 2014), dark rearing (Duffy and Mitchell, 2013), and various pharmacological interventions (Morishita et al., 2010; Nabel and Morishita, 2013; Hübener and Bonhoeffer, 2014; Sadahiro et al., 2017). 
Together, the present findings reveal that transplant-induced plasticity is not merely the result of a parallel circuit of transplanted cells but rather is a property of the host network that is induced but not maintained by the transplanted cells. How the small number of transplanted neurons induces the second $\mathrm{CP}$ remains to be understood. One possible explanation consistent with theoretical studies of attractor networks (Aljadeff et al., 2015 ) is that initial random connections of the transplanted neurons perturb the stability of the cortical network, allowing the relatively weak stimulus produced by monocular occlusion to push it out of its attractor state. The destabilized network could then rewire and settle into a new attractor state in which only one eye was providing strong excitation. Once settled into the new state, it would again become refractory to change. Future work will be required to determine anatomical and functional changes in the synaptic connections.

\section{References}

Aljadeff J, Stern M, Sharpee T (2015) Transition to chaos in random networks with cell-type-specific connectivity. Phys Rev Lett 114:088101.

Atencio CA, Schreiner CE (2008) Spectrotemporal processing differences between auditory cortical fast-spiking and regular-spiking neurons. J Neurosci 28:3897-3910.

Balog J, Matthies U, Naumann L, Voget M, Winter C, Lehmann K (2014) Social experience modulates ocular dominance plasticity differentially in adult male and female mice. Neuroimage 103:454-461.

Barthó P, Hirase H, Monconduit L, Zugaro M, Harris KD, Buzsáki G (2004) Characterization of neocortical principal cells and interneurons by network interactions and extracellular features. J Neurophysiol 92:600-608.

Benjamini Y, Hochberg Y (1995) Controlling the false discovery rate: a practical and powerful approach to multiple testing. J R Stat Soc 57: 289-300.

Brainard DH (1997) The psychophysics toolbox. Spat Vis 10:433-436.

Bukalo O, Pinard CR, Silverstein S, Brehm C, Hartley ND, Whittle N, Colacicco G, Busch E, Patel S, Singewald N, Holmes A (2015) Prefrontal inputs to the amygdala instruct fear extinction memory formation. Sci Adv 1:e1500251.

Cang J, Kalatsky VA, Löwel S, Stryker MP (2005) Optical imaging of the intrinsic signal as a measure of cortical plasticity in the mouse. Vis Neurosci 22:685-691.

Cardin JA (2012) Dissecting local circuits in vivo: integrated optogenetic and electrophysiology approaches for exploring inhibitory regulation of cortical activity. J Physiol Paris 106:104-111.

Chung JE, Magland JF, Barnett AH, Tolosa VM, Tooker AC, Lee KY, Shah KG, Felix SH, Frank LM, Greengard LF (2017) A fully automated approach to spike sorting. Neuron 95:1381-1394.e6.

Chuong AS, Miri ML, Busskamp V, Matthews GA, Acker LC, Sørensen AT, Young A, Klapoetke NC, Henninger MA, Kodandaramaiah SB, Ogawa M, Ramanlal SB, Bandler RC, Allen BD, Forest CR, Chow BY, Han X, Lin Y, Tye KM, Roska B, Cardin JA, et al. (2014) Noninvasive optical inhibition with a red-shifted microbial rhodopsin. Nat Neurosci 17:1123-1129.

Connors BW, Kriegstein AR (1986) Cellular physiology of the turtle visual cortex: distinctive properties of pyramidal and stellate neurons. J Neurosci 6:164-177.

Davis MF, Figueroa Velez DX, Guevarra RP, Yang MC, Habeeb M, Carathedathu MC, Gandhi SP (2015) Inhibitory neuron transplantation into adult visual cortex creates a new critical period that rescues impaired vision. Neuron 86:1055-1066.

Du J, Blanche TJ, Harrison RR, Lester HA, Masmanidis SC (2011) Multiplexed, high density electrophysiology with nanofabricated neural probes. PLoS One 6:e26204.

Duffy KR, Mitchell DE (2013) Darkness alters maturation of visual cortex and promotes fast recovery from monocular deprivation. Curr Biol 23: 382-386.

Espinosa JS, Stryker MP (2012) Development and plasticity of the primary visual cortex. Neuron 75:230-249.

Fagiolini M, Hensch TK (2000) Inhibitory threshold for critical-period activation in primary visual cortex. Nature 404:183-186.

Feldman DE (2000) Inhibition and plasticity. Nat Neurosci 3:303-304.

Figueroa Velez DX, Ellefsen KL, Hathaway ER, Carathedathu MC, Gandhi SP
(2017) Contribution of innate cortical mechanisms to the maturation of orientation selectivity in parvalbumin interneurons. J Neurosci 37: $820-829$.

Fukunaga I, Herb JT, Kollo M, Boyden ES, Schaefer AT (2014) Independent control of gamma and theta activity by distinct interneuron networks in the olfactory bulb. Nat Neurosci 17:1208-1216.

Gandhi SP, Yanagawa Y, Stryker MP (2008) Delayed plasticity of inhibitory neurons in developing visual cortex. Proc Natl Acad Sci U S A 105: 16797-16802.

Greifzu F, Pielecka-Fortuna J, Kalogeraki E, Krempler K, Favaro PD, Schlüter OM, Löwel S (2014) Environmental enrichment extends ocular dominance plasticity into adulthood and protects from stroke-induced impairments of plasticity. Proc Natl Acad Sci U S A 111:1150-1155.

Han X, Chow BY, Zhou H, Klapoetke NC, Chuong A, Rajimehr R, Yang A, Baratta MV, Winkle J, Desimone R, Boyden ES (2011) A high-light sensitivity optical neural silencer: development and application to optogenetic control of non-human primate cortex. Front Syst Neurosci 5:18.

Hanover JL, Huang ZJ, Tonegawa S, Stryker MP (1999) Brain-derived neurotrophic factor overexpression induces precocious critical period in mouse visual cortex. J Neurosci 19:RC40.

Hengen KB, Lambo ME, Van Hooser SD, Katz DB, Turrigiano GG (2013) Firing rate homeostasis in visual cortex of freely behaving rodents. Neuron 80:335-342.

Hensch TK (2005) Critical period plasticity in local cortical circuits. Nat Rev Neurosci 6:877-888.

Hensch TK, Fagiolini M, Mataga N, Stryker MP, Baekkeskov S, Kash SF (1998) Local GABA circuit control of experience-dependent plasticity in developing visual cortex. Science 282:1504-1508.

Hofer SB, Mrsic-Flogel TD, Bonhoeffer T, Hübener M (2006) Prior experience enhances plasticity in adult visual cortex. Nat Neurosci 9:127-132.

Hübener M, Bonhoeffer T (2014) Neuronal plasticity: beyond the critical period. Cell 159:727-737.

Kaneko M, Stryker MP (2014) Sensory experience during locomotion promotes recovery of function in adult visual cortex. Elife 3:e02798.

Khibnik LA, Cho KK, Bear MF (2010) Relative contribution of feedforward excitatory connections to expression of ocular dominance plasticity in layer 4 of visual cortex. Neuron 66:493-500.

Kleiner M, Brainard DH, Pelli DG (2007) What's new in Psychtoolbox-3? Perception 36:14.

Kuhlman SJ, Tring E, Trachtenberg JT (2011) Fast-spiking interneurons have an initial orientation bias that is lost with vision. Nat Neurosci 14: $1121-1133$

Kuhlman SJ, Olivas ND, Tring E, Ikrar T, Xu X, Trachtenberg JT (2013) A disinhibitory microcircuit initiates critical-period plasticity in the visual cortex. Nature 501:543-546.

Larimer P, Spatazza J, Espinosa JS, Tang Y, Kaneko M, Hasenstaub AR, Stryker MP, Alvarez-Buylla A (2016) Caudal ganglionic eminence precursor transplants disperse and integrate as lineage-specific interneurons but do not induce cortical plasticity. Cell Rep 16:1391-1404.

Madisen L, et al. (2012) A toolbox of cre-dependent optogenetic transgenic mice for light-induced activation and silencing. Nat Neurosci 15:793802.

Moore AK, Weible AP, Balmer TS, Trussell LO, Wehr M (2018) Rapid rebalancing of excitation and inhibition by cortical circuitry. Neuron 97 : 1341-1355.e6.

Morishita H, Miwa JM, Heintz N, Hensch TK (2010) Lynxl, a cholinergic brake, limits plasticity in adult visual cortex. Science 330:1238-1240.

Nabel EM, Morishita H (2013) Regulating critical period plasticity: insight from the visual system to fear circuitry for therapeutic interventions. Front Psychiatry 4:146.

Niell CM, Stryker MP (2008) Highly selective receptive fields in mouse visual cortex. J Neurosci 28:7520-7536.

Phillips EA, Hasenstaub AR (2016) Asymmetric effects of activating and inactivating cortical interneurons. Elife 5:e18383.

Pizzorusso T, Medini P, Berardi N, Chierzi S, Fawcett JW, Maffei L (2002) Reactivation of ocular dominance plasticity in the adult visual cortex. Science 298:1248-1251.

Priya R, Rakela B, Kaneko M, Spatazza J, Larimer P, Hoseini MS, Hasenstaub AR, Alvarez-Buylla A, Stryker MP (2019) Vesicular GABA transporter is necessary for transplant-induced critical period plasticity in mouse visual cortex. J Neurosci 39:2635-2648.

Sadahiro M, Demars MP, Burman P, Yevoo P, Smith MR, Zimmer A, Morishita 
H (2017) Activation of somatostatin inhibitory neurons by Lypd6$\mathrm{nAChR} \alpha 2$ system restores juvenile-like plasticity in adult visual cortex. Available from: https://www.biorxiv.org/content/10.1101/155465v1.

Sadeh S, Silver RA, Mrsic-Flogel TD, Muir DR (2017) Assessing the role of inhibition in stabilizing neocortical networks requires large-scale perturbation of the inhibitory population. J Neurosci 37:12050-12067.

Saiepour MH, Rajendran R, Omrani A, Ma WP, Tao HW, Heimel JA, Levelt CN (2015) Ocular dominance plasticity disrupts binocular inhibitionexcitation matching in visual cortex. Curr Biol 25:713-721.

Sato M, Stryker MP (2008) Distinctive features of adult ocular dominance plasticity. J Neurosci 28:10278-10286.

Sawtell NB, Frenkel MY, Philpot BD, Nakazawa K, Tonegawa S, Bear MF (2003) NMDA receptor-dependent ocular dominance plasticity in adult visual cortex. Neuron 38:977-985.

Southwell DG, Froemke RC, Alvarez-Buylla A, Stryker MP, Gandhi SP (2010) Cortical plasticity induced by inhibitory neuron transplantation. Science 327:1145-1148.
Tang Y, Stryker MP, Alvarez-Buylla A, Espinosa JS (2014) Cortical plasticity induced by transplantation of embryonic somatostatin or parvalbumin interneurons. Proc Natl Acad Sci U S A 111:18339-18344.

Troyer TW, Krukowski AE, Priebe NJ, Miller KD (1998) Contrastinvariant orientation tuning in cat visual cortex: thalamocortical input tuning and correlation-based intracortical connectivity. J Neurosci 18:5908-5927.

Vogt D, Wu PR, Sorrells SF, Arnold C, Alvarez-Buylla A, Rubenstein JL (2015) Viral-mediated labeling and transplantation of medial ganglionic eminence (MGE) cells for in vivo studies. J Vis Exp 98:52740.

Wang BS, Sarnaik R, Cang J (2010) Critical period plasticity matches binocular orientation preference in the visual cortex. Neuron 65:246-256.

Wiesel TN, Hubel DH (1963) Single-cell responses in striate cortex of kittens deprived of vision in one eye. J Neurophysiol 26:1003-1017.

Yaeger CE, Ringach DL, Trachtenberg JT (2019) Neuromodulatory control of localized dendritic spiking in critical period cortex. Nature 567:100104 . 